\title{
A CLASS OF SLOWLY CONVERGENT SERIES AND THEIR SUMMATION BY GAUSSIAN QUADRATURE
}

\author{
WALTER GAUTSCHI
}

\begin{abstract}
Series are considered whose general term is a rational function multiplied by a fractional power. The summation of such series is reduced, via Laplace transformation techniques, to a problem of quadrature, which is then solved by Gaussian quadrature relative to Einstein and Fermi weight functions. A number of examples are worked out in detail.
\end{abstract}

\section{INTRODUCTION}

We consider series of the type

$$
S_{0}=\sum_{k=1}^{\infty} k^{\nu-1} r(k)
$$

or

$$
S_{1}=\sum_{k=1}^{\infty}(-1)^{k-1} k^{\nu-1} r(k),
$$

where $0<\nu \leq 1$ and $r(\cdot)$ is a rational function

$$
r(s)=\frac{p(s)}{q(s)}
$$

with $p, q$ real polynomials of $\operatorname{degrees} \operatorname{deg} p \leq \operatorname{deg} q$. Strict inequality is assumed when necessary for convergence. It is further assumed that the zeros of $q$ all have nonpositive real parts:

$$
\text { if } q(-a)=0 \text { then } \operatorname{Re} a \geq 0 \text {. }
$$

This condition can always be achieved by a preliminary summation of a few initial terms.

The problem can be simplified by first obtaining the partial fraction decomposition of $r$,

$$
r(s)=\sum_{\rho} \sum_{m=1}^{m_{\rho}} c_{\rho m}\left(s+a_{\rho}\right)^{-m}+\sum_{\gamma} \sum_{m=1}^{m_{\gamma}}\left[c_{\gamma m}\left(s+a_{\gamma}\right)^{-m}+\bar{c}_{\gamma m}\left(s+\bar{a}_{\gamma}\right)^{-m}\right],
$$

Received March 7, 1990; revised September 17, 1990.

1980 Mathematics Subject Classification (1985 Revision). Primary 40A25; Secondary 44A10, 65D30, 33A65.

Key words and phrases. Slowly convergent series, Laplace transformation, summation by quadrature, Gaussian quadrature, orthogonal polynomials.

Work supported, in part, by the National Science Foundation under grant CCR-8704404. 
where the first sum is over all real zeros $\left(-a_{\rho}\right)$ of $q$ (with multiplicities $\left.m_{\rho}\right)$, and the second sum is over all pairs of conjugate complex zeros $\left(-a_{\gamma},-\bar{a}_{\gamma}\right)$ (with multiplicities $m_{\gamma}$ ). The coefficients $c_{\rho m}$ in the first sum are real, those in the second complex, in general. (We have assumed in (1.4) that $\operatorname{deg} p<$ $\operatorname{deg} q$. If $\operatorname{deg} p=\operatorname{deg} q$, and we are thus dealing with $S_{1}$ in (1.1), there will be an additional constant term in (1.4). Its contribution to the series in (1.1) is expressible in terms of the Riemann zeta function; cf. (3.9) below for $m=$ 0 .) Once the decomposition (1.4) has been obtained (for relevant constructive methods, see, e.g., $[8, \S 7.1])$, it clearly suffices to consider

$$
r(s)=\frac{1}{(s+a)^{m}}, \quad \operatorname{Re} a \geq 0, m \geq 1 .
$$

Without restriction of generality, it may be further assumed that $\operatorname{Im} a \geq 0$.

By interpreting the terms in the series (1.0) and (1.1) as Laplace transforms at integer values, it is possible to express the sum of the series as a weighted integral over $\mathbb{R}_{+}$of certain special functions related to the incomplete gamma function. The weighting involves the product of a fractional power and either Einstein's function $t\left(e^{t}-1\right)^{-1}$ (in the case of (1.0)), or Fermi's function $\left(e^{t}+1\right)^{-1}$ (in the case of (1.1)), both having infinitely many poles on the imaginary axis of the complex plane. Properties of the required special functions are briefly developed in $\S 2$. Section 3 discusses the summation of (1.0), (1.1) via Gaussian quadrature. The case $\nu=1$ of purely rational series is treated in $\S 4$ and complements more traditional techniques (e.g., those in $[8, \S 7.2 \mathrm{II}])$. Numerical examples for the case $\nu=\frac{1}{2}$ are given in $\S 5$, where also comparisons are made with direct summation and accelerated summation using the $\varepsilon$-algorithm.

\section{Preliminaries}

Define, for $t>0$,

$$
g_{-1}(t ; \nu)=g_{-1}(t)=\frac{t^{-1}}{\Gamma(1-\nu)}, \quad 0<\nu<1,
$$

and for $t>0, n=0,1,2, \ldots$,

$$
g_{n}(t ; a, \nu)=g_{n}(t)=\frac{e^{-a t} t^{\nu-1}}{n ! \Gamma(1-\nu)} \int_{0}^{t} e^{a \tau}(t-\tau)^{n} \tau^{-\nu} d \tau,
$$

$$
\operatorname{Re} a \geq 0, \quad \operatorname{Im} a \geq 0, \quad a \neq 0, \quad 0<\nu<1 .
$$

Lemma 2.1. We have

$$
g_{0}(t ; a, \nu)=e^{-a t} \gamma^{*}(1-\nu,-a t),
$$

where $\gamma^{*}$ is Tricomi's form of the incomplete gamma function (cf. [3, eq. 6.5.4]). Furthermore,

$$
\begin{aligned}
& g_{n+1}(t)=\frac{1}{n+1}\left\{\left(t+\frac{n+1-\nu}{a}\right) g_{n}(t)-\frac{t}{a} g_{n-1}(t)\right\} \\
& n=0,1,2, \ldots .
\end{aligned}
$$


Proof. By definition,

$$
g_{0}(t)=\frac{e^{-a t} t^{\nu-1}}{\Gamma(1-\nu)} \int_{0}^{t} e^{a \tau} \tau^{-\nu} d \tau
$$

which, upon the change of variables $u=-a \tau$, gives

$$
\begin{aligned}
g_{0}(t) & =\frac{e^{-a t}(-a t)^{\nu-1}}{\Gamma(1-\nu)} \int_{0}^{-a t} e^{-u} u^{-\nu} d u \\
& =\frac{e^{-a t}(-a t)^{-(1-\nu)}}{\Gamma(1-\nu)} \gamma(1-\nu,-a t)=e^{-a t} \gamma^{*}(1-\nu,-a t)
\end{aligned}
$$

(cf. [3, eqs. 6.5.2, 6.5.4]). Next,

$$
\begin{aligned}
g_{n+1}(t) & =\frac{e^{-a t} t^{\nu-1}}{(n+1) ! \Gamma(1-\nu)} \int_{0}^{t} e^{a \tau}(t-\tau)^{n}(t-\tau) \tau^{-\nu} d \tau \\
& =\frac{t}{n+1} g_{n}(t)-\frac{e^{-a t} t^{\nu-1}}{(n+1) ! \Gamma(1-\nu)} \int_{0}^{t} e^{a \tau}(t-\tau)^{n} \tau^{1-\nu} d \tau .
\end{aligned}
$$

To the last integral we apply integration by parts, letting $u=\tau^{1-\nu}(t-\tau)^{n}$, $v^{\prime}=e^{a \tau}$, hence

$$
\begin{aligned}
u^{\prime} & =(1-\nu) \tau^{-\nu}(t-\tau)^{n}-n \tau^{1-\nu}(t-\tau)^{n-1} \\
& =(n+1-\nu)(t-\tau)^{n} \tau^{-\nu}-n t(t-\tau)^{n-1} \tau^{-\nu}, \\
v & =\frac{1}{a} e^{a \tau} .
\end{aligned}
$$

This yields (2.3) for $n \geq 1$. A similar calculation gives

$$
g_{1}(t)=\left(t+\frac{1-\nu}{a}\right) g_{0}(t)-\frac{1}{a \Gamma(1-\nu)},
$$

which, in view of $\left(2.1_{-1}\right)$, shows that $(2.3)$ holds also for $n=0$.

To the author's knowledge, no software seems to be readily available for computing the incomplete gamma function in the domain of interest here (left half plane), and one thus has to rely on standard techniques such as power series and asymptotic expansions. An effort of developing good software for Tricomi's function $\gamma^{*}(1-\nu, z), 0<\nu<1$, applicable for arbitrary complex $z$, would certainly be worthwhile. For the case $\nu=\frac{1}{2}$, however, see $\S 5$.

\section{Summation of $S_{0}$ and $S_{1}$}

We employ a technique already used in [7], namely, to interpret the general term of the series as a Laplace transform and thereby converting the series into a suitably weighted integral. We first treat the series $S_{0}$ in $(1.0)$.

Assume $r(\cdot)$ given as in (1.5), and consider first the case $a \neq 0$. Then

$$
s^{\nu-1} \cdot \frac{1}{(s+a)^{m}}=\mathscr{L}\left\{\frac{t^{-\nu}}{\Gamma(1-\nu)} * \frac{t^{m-1} e^{-a t}}{(m-1) !}\right\}, \quad 0<\nu<1,
$$


where $\mathscr{L}\{\cdot\}$ is the Laplace transform and * means convolution. We thus have

$$
\begin{aligned}
f(t) & :=\mathscr{L}^{-1}\left\{\frac{s^{\nu-1}}{(s+a)^{m}}\right\}=\frac{1}{(m-1) ! \Gamma(1-\nu)} \int_{0}^{t} e^{-a(t-\tau)}(t-\tau)^{m-1} \tau^{-\nu} d \tau \\
(3.2) & =\frac{e^{-a t}}{(m-1) ! \Gamma(1-\nu)} \int_{0}^{t} e^{a \tau}(t-\tau)^{m-1} \tau^{-\nu} d \tau \\
& =t^{1-\nu} g_{m-1}(t ; a, \nu), \quad m \geq 1,
\end{aligned}
$$

where $g_{n}(t ; a, \nu)$ is defined in $\left(2.1_{n}\right)$. There follows

$$
\begin{aligned}
\sum_{k=1}^{\infty} \frac{k^{\nu-1}}{(k+a)^{m}} & =\sum_{k=1}^{\infty}(\mathscr{L} f)(k)=\sum_{k=1}^{\infty} \int_{0}^{\infty} e^{-k t} f(t) d t \\
& =\sum_{k=1}^{\infty} \int_{0}^{\infty} e^{-t} t^{1-\nu} e^{-(k-1) t} g_{m-1}(t ; a, \nu) d t \\
& =\int_{0}^{\infty} e^{-t} t^{1-\nu} \sum_{k=1}^{\infty} e^{-(k-1) t} g_{m-1}(t ; a, \nu) d t \\
& =\int_{0}^{\infty} t^{1-\nu} \frac{1}{e^{t}-1} g_{m-1}(t ; a, \nu) d t .
\end{aligned}
$$

Thus,

$$
\sum_{k=1}^{\infty} \frac{k^{\nu-1}}{(k+a)^{m}}=\int_{0}^{\infty} t^{-\nu} \varepsilon(t) g_{m-1}(t ; a, \nu) d t, \quad m \geq 1,0<\nu<1,
$$

where $\varepsilon(\cdot)$ is "Einstein's function" (cf. [7]),

$$
\varepsilon(t)=\frac{t}{e^{t}-1}, \quad 0 \leq t<\infty .
$$

Since $g_{m-1}(\cdot ; a, \nu)$, by Lemma 2.1 , is an entire function, formula (3.3) suggests to apply Gaussian quadrature to the integral on the right, using $w(t)=$ $t^{-\nu} \varepsilon(t)$ as a weight function on $[0, \infty]$. The required orthogonal polynomials can be computed by techniques already discussed in [7] (see, in particular, (2.3) and Example 4.4 of that paper). Gauss quadrature will converge quite rapidly, unless $\operatorname{Re} a$ and/or $\operatorname{Im} a$ is large, in which case "stratified" summation can be employed to regain rapid convergence (see Examples 5.1 and 5.4). For $\nu=\frac{1}{2}$, the first 80 recursion coefficients for the required orthogonal polynomials are given to 25 significant digits in Table 1 of the Appendix.

It is easily seen that (3.3) holds also for $a=0$ if we define

$$
\begin{array}{r}
g_{0}(t)=\frac{1}{\Gamma(2-\nu)}, \quad g_{n+1}(t)=\frac{t}{n+2-\nu} g_{n}(t), \\
n=0,1,2, \ldots(a=0) .
\end{array}
$$

The series can then be expressed in terms of Riemann's zeta function,

$$
\sum_{k=1}^{\infty} k^{-(m+1-\nu)}=\zeta(m+1-\nu),
$$


and since $g_{m-1}$ is a monomial of degree $m-1$, the $n$-point Gauss formula for the integral in (3.3) gives exact answers (modulo rounding) if $n=\lfloor(m+1) / 2\rfloor$.

For the sum $S_{1}$ in (1.1), a calculation similar to the one which led to (3.3) now yields, for $a \neq 0$,

$$
\begin{aligned}
& \sum_{k=1}^{\infty}(-1)^{k-1} \frac{k^{\nu-1}}{(k+a)^{m}}=\int_{0}^{\infty} t^{-\nu} \varphi(t) \cdot \operatorname{tg}_{m-1}(t ; a, \nu) d t \\
& m \geq 0,0<\nu<1,
\end{aligned}
$$

where $\varphi(\cdot)$ is the "Fermi weight function" (cf. [7]),

$$
\varphi(t)=\frac{1}{e^{t}+1}, \quad 0 \leq t<\infty .
$$

The result (3.7), as noted, holds also for $m=0$, if $g_{-1}$ is defined as in $\left(2.1_{-1}\right)$. If $a=0$, then (3.7) holds with $g_{m-1}, m \geq 0$, defined in $\left(2.1_{-1}\right)$ and (3.5), and represents the series

$$
\sum_{k=1}^{\infty}(-1)^{k-1} k^{-(m+1-\nu)}=\left(1-2^{-m+\nu}\right) \zeta(m+1-\nu) .
$$

Again, Gauss quadrature for the integral in (3.7), in this case, is exact if we take $n=\lfloor(m+2) / 2\rfloor$ points.

The first 80 recursion coefficients for the orthogonal polynomials with respect to the weight function $t^{-\nu} \varphi(t), \nu=\frac{1}{2}$, are listed in Table 2 of the Appendix.

Remarks. 1. The fractional power $k^{\nu-1}$ in (1.0), (1.1) can easily be generalized to $(k+b)^{\nu-1}, \operatorname{Re} b \geq 0$, since this only introduces a factor $e^{-b t}$ in $(3.1)$ and gives $f(t)=t^{1-\nu} e^{-b t} g_{m-1}(t ; a-b, \nu)$ in place of (3.2), hence

$$
\sum_{k=1}^{\infty} \frac{(k+b)^{\nu-1}}{(k+a)^{m}}=\int_{0}^{\infty} t^{-\nu} \varepsilon(t) e^{-b t} g_{m-1}(t ; a-b, \nu) d t,
$$

and the similarly generalized version of (3.7),

$$
\sum_{k=1}^{\infty}(-1)^{k-1} \frac{(k+b)^{\nu-1}}{(k+a)^{m}}=\int_{0}^{\infty} t^{-\nu} \varphi(t) \cdot t e^{-b t} g_{m-1}(t ; a-b, \nu) d t .
$$

2. As an alternative to Gauss quadrature with weight function $t^{-\nu} \varepsilon(t)$, one could write the integral in (3.3) as

$$
\int_{0}^{\infty} t^{-\nu} \varepsilon(t) g_{m-1}(t ; a, \nu) d t=\int_{0}^{\infty} t^{-\nu} e^{-t} \cdot \frac{t}{1-e^{-t}} g_{m-1}(t ; a, \nu) d t
$$

and apply Gauss-Laguerre quadrature to the integral on the right. The poles that were previously incorporated in the weight function $t^{-\nu} \varepsilon(t)$ then, however, become part of the integrand, which may adversely affect the speed of convergence of the quadrature scheme. This was indeed found to be the case when $a$ is relatively small, but for larger values of $a$, in particular when used 
in conjunction with stratified summation (cf. Examples 5.1 and 5.4), the GaussLaguerre method is competitive and, in Example 5.1, even more efficient.

The same alternative approach is possible for the integral in (3.7), if written as

$$
\int_{0}^{\infty} t^{-\nu} \varphi(t) \cdot t g_{m-1}(t ; a, \nu) d t=\int_{0}^{\infty} t^{-\nu} e^{-t} \cdot \frac{t}{1+e^{-t}} g_{m-1}(t ; a, \nu) d t
$$

although this time the poles affect convergence more severely, since they are half as close to the real axis than before. Still, for sufficiently large values of $a$, Gauss-Laguerre quadrature here, too, gains the upper hand.

Finally, if many different values of $\nu$ were contemplated, then GaussLaguerre would also be preferable, since the recursion coefficients for the respective orthogonal polynomials-the generalized Laguerre polynomials-are then explicitly known and need not be tabulated. In the case of the weight function $w(t)=t^{-\nu} e^{-t}$ on $(0, \infty)$, the corresponding (monic) orthogonal polynomials $\pi_{k}(\cdot)=\pi_{k}(\cdot ; w)$ indeed satisfy

$$
\begin{gathered}
\pi_{k+1}(t)=\left(t-\alpha_{k}\right) \pi_{k}(t)-\beta_{k} \pi_{k-1}(t), \quad k=0,1,2, \ldots, \\
\pi_{-1}(t)=0, \quad \pi_{0}(t)=1,
\end{gathered}
$$

with the coefficients $\alpha_{k}=\alpha_{k}(w), \beta_{k}=\beta_{k}(w)\left[\beta_{0}(w)=\int_{0}^{\infty} w(t) d t\right]$ having the particularly simple form

$$
\begin{gathered}
\alpha_{k}(w)=2 k+1-\nu, \quad k \geq 0 ; \\
\beta_{0}(w)=\Gamma(1-\nu), \quad \beta_{k}(w)=k(k-\nu), \quad k \geq 1
\end{gathered} \quad\left(w=t^{-\nu} e^{-t}\right) .
$$

\section{SERIES OF PURELY RATIONAL TERMS}

So far, we assumed that $0<\nu<1$ in (1.0) and (1.1). The same techniques, however, are applicable when $\nu=1$, i.e., for series

$$
S_{0}^{\prime}=\sum_{k=1}^{\infty} r(k), \quad S_{1}^{\prime}=\sum_{k=1}^{\infty}(-1)^{k-1} r(k) .
$$

One finds, when

$$
r(s)=\frac{1}{(s+a)^{m}}, \quad \operatorname{Re} a \geq 0
$$

for $m \geq 2$ that

$$
\sum_{k=1}^{\infty} \frac{1}{(k+a)^{m}}=\frac{1}{(m-1) !} \int_{0}^{\infty} \varepsilon(t) t^{m-2} e^{-a t} d t,
$$

and for $m \geq 1$ that

$$
\sum_{k=1}^{\infty} \frac{(-1)^{k-1}}{(k+a)^{m}}=\frac{1}{(m-1) !} \int_{0}^{\infty} \varphi(t) t^{m-1} e^{-a t} d t,
$$

with $\varepsilon$ and $\varphi$ as given in (3.4) and (3.8), respectively. 
For more general rational functions

$$
r(s)=\sum_{i} \sum_{m=1}^{m_{i}} c_{i m}\left(s+a_{i}\right)^{-m}
$$

we get from (4.3)

$$
\sum_{k=1}^{\infty} r(k)=\int_{0}^{\infty} \varepsilon(t) g(t) d t
$$

where

$$
g(t)=\sum_{i} \sum_{m=1}^{m_{i}} \frac{c_{i m}}{(m-1) !} t^{m-2} e^{-a_{i} t}
$$

Evidently, $g$ is again an entire function, provided

$$
\sum_{i} c_{i 1}=0
$$

which is required for the series in (4.6) to converge. Similarly,

$$
\sum_{k=1}^{\infty}(-1)^{k-1} r(k)=\int_{0}^{\infty} \varphi(t) h(t) d t
$$

where

$$
h(t)=\sum_{i} \sum_{m=1}^{m_{i}} \frac{c_{i m}}{(m-1) !} t^{m-1} e^{-a_{i} t},
$$

an entire function for any choice of the coefficients $c_{i m}$.

The first 40 recursion coefficients for the polynomials orthogonal with respect to $\varepsilon$ and $\varphi$ can be found to 25 significant digits in [7, Appendices A1 and A2].

The method described in this section provides an alternative to other summation/integration methods, such as those discussed in [8, §7.2II]. An advantage of the present method is that it leads to Gaussian quadrature of entire functions, a possible complication, that the interval of integration is infinite.

\section{EXAMPLES}

In all of our examples we take $\nu=\frac{1}{2}$. In this case, the function $g_{0}$ in (2.2) is given by (cf. [3, eq. 6.5.18])

$$
g_{0}\left(t ; a, \frac{1}{2}\right)=e^{-a t} \gamma^{*}\left(\frac{1}{2},-a t\right)=\frac{2}{\sqrt{\pi}} \frac{F(\sqrt{a t})}{\sqrt{a t}},
$$

where $F$ is Dawson's integral,

$$
F(z)=e^{-z^{2}} \int_{0}^{z} e^{t^{2}} d t
$$

For real $z$, this can be evaluated with an accuracy of up to about 20 significant digits, using the rational Chebyshev approximations given in [2].

All computations reported below were done in double precision on the Cyber 205 computer (the equivalent of about 29 decimal places).

Example 5.1. $S_{0}=\sum_{k=1}^{\infty} k^{-1 / 2} /(k+a)^{m}$. 
This series with $a=m=1$ was communicated to the author by Professor P. J. Davis, who encountered it in his study of spirals [4].

We computed $S_{0}$ from (3.3) (with $\left.\nu=\frac{1}{2}\right),(5.1)$, and $\left(2.1_{-1}\right),(2.3)$, for $a=.5,1 ., 2 ., 4 ., 8$. and $m=1(1) 5$. The integral in (3.3) was evaluated by $n$-point Gaussian quadrature rules, which were generated with the help of the recursion coefficients in Table 1 of the Appendix and well-known eigenvalue techniques (see, e.g., [6, $\S 1.3(\mathrm{iv})]$ ). In Table 5.1 we show only the results for $m=1$; those for $m>1$ are similar, but exhibit somewhat slower convergence. It is evident that, as $a$ increases, convergence of the Gauss quadrature formula slows down considerably. The reason for this is the behavior of the function $g_{0}$ on the right of (5.1), which for increasing $a$ approaches a discontinuous function (see Figure 5.1).

TABLE 5.1

n-point quadrature approximations to the integral in (3.3) with $\nu=\frac{1}{2}, m=1, a=.5,1 ., 2 ., 4 ., 8$.

\begin{tabular}{r|lll}
\hline \multicolumn{1}{c|}{$n$} & \multicolumn{1}{c}{$a=.5$} & \multicolumn{1}{c}{$a=1}$. & \multicolumn{1}{c}{$a=2}$. \\
\hline 5 & 2.1344163 & 1.8599 & 1.537 \\
10 & 2.1344166429861 & 1.860025078 & 1.53967 \\
15 & 2.1344166429862372611 & 1.86002507922117 & 1.539680509 \\
20 & 2.1344166429862372611 & 1.860025079221190306 & 1.539680512350 \\
25 & & 1.8600250792211903071 & 1.53968051235329 \\
30 & & 1.8600250792211903072 & 1.539680512353302010 \\
35 & & & 1.5396805123533020128 \\
40 & & & 1.5396805123533020128 \\
\hline
\end{tabular}

\begin{tabular}{r|ll}
\hline$n$ & \multicolumn{1}{c}{$a=4}$. & \multicolumn{1}{c}{$a=8}$. \\
\hline 5 & 1.19 & .8 \\
10 & 1.217 & .91 \\
15 & 1.21826 & .930 \\
20 & 1.218273 & .9312 \\
25 & 1.218274011 & .93135 \\
30 & 1.21827401461 & .931371 \\
35 & 1.218274014668 & .9313727 \\
40 & 1.2182740146698 & .93137291 \\
\hline
\end{tabular}

To achieve better accuracy, when $a$ is large, we proceed as follows. With $a_{0}=\lfloor a\rfloor$ denoting the largest integer $\leq a$, and $a=a_{0}+a_{1}$, where $a_{0} \geq 1$, $0 \leq a_{1}<1$, the summation over all $k \geq 1$ may be "stratified" by letting $k=\lambda+\kappa a_{0}$ and summing over all $\kappa \geq 0$ for $\lambda=1,2, \ldots, a_{0}$. Thus,

$$
\begin{aligned}
S_{0} & =\sum_{k=1}^{\infty} \frac{k^{-1 / 2}}{\left(k+a_{0}+a_{1}\right)^{m}}=\sum_{\lambda=1}^{a_{0}} \sum_{\kappa=0}^{\infty} \frac{\left(\lambda+\kappa a_{0}\right)^{-1 / 2}}{\left(\lambda+\kappa a_{0}+a_{0}+a_{1}\right)^{m}} \\
& =a_{0}^{-(m+1 / 2)} \sum_{\lambda=1}^{a_{0}} \sum_{\kappa=0}^{\infty} \frac{\left(\kappa+\lambda / a_{0}\right)^{-1 / 2}}{\left(\kappa+1+\left(\lambda+a_{1}\right) / a_{0}\right)^{m}} \\
& =a_{0}^{-(m+1 / 2)} \sum_{\lambda=1}^{a_{0}}\left\{\sum_{\kappa=1}^{\infty} \frac{\left(\kappa+\lambda / a_{0}\right)^{-1 / 2}}{\left(\kappa+1+\left(\lambda+a_{1}\right) / a_{0}\right)^{m}}+\frac{\left(\lambda / a_{0}\right)^{-1 / 2}}{\left(1+\left(\lambda+a_{1}\right) / a_{0}\right)^{m}}\right\} .
\end{aligned}
$$




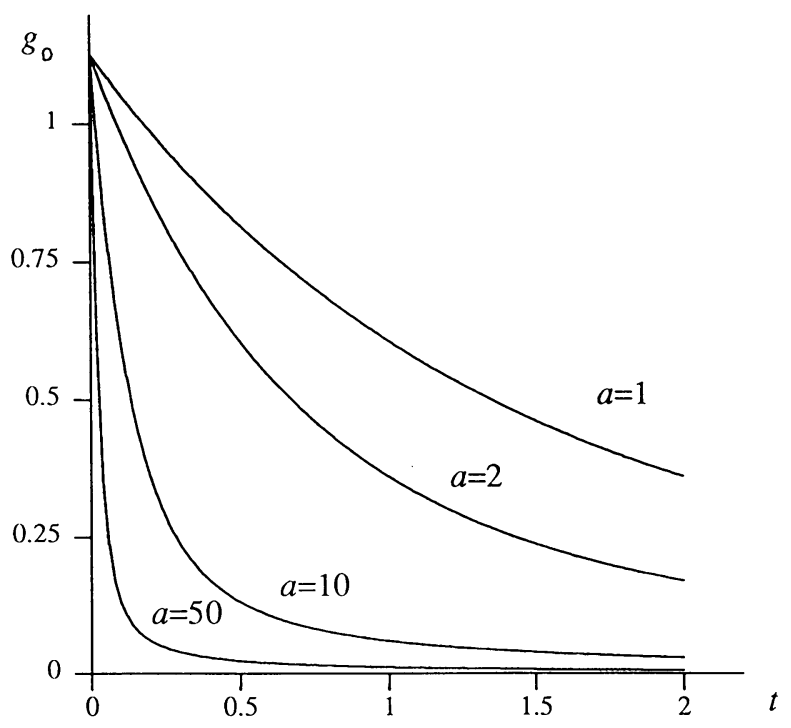

FIGURE 5.1

The function $g_{0}$ in (5.1)

To the inner sum we now apply (3.10) to obtain

$$
\begin{aligned}
& \sum_{\kappa=1}^{\infty} \frac{\left(\kappa+\lambda / a_{0}\right)^{-1 / 2}}{\left(\kappa+1+\left(\lambda+a_{1}\right) / a_{0}\right)^{m}} \\
& \quad=\int_{0}^{\infty} t^{-1 / 2} \varepsilon(t) e^{-\left(\lambda / a_{0}\right) t} g_{m-1}\left(t ; 1+\frac{a_{1}}{a_{0}}, \frac{1}{2}\right) d t .
\end{aligned}
$$

The "effective" parameter in $g_{m-1}$ is now $1+a_{1} / a_{0}$, a number close to 1 , and the coefficient $\lambda / a_{0}$ in the exponential is bounded by 1 . Gauss quadrature applied to the integral in (5.4) should therefore converge quite rapidly, indeed, more so the larger $a_{0}$ ! This is borne out by the results displayed for $m=1$ and $a=a_{0}=8 ., 16 ., 32$. in Table 5.2.

The correct number of significant decimal digits produced by direct summation of the series, using 1000 terms, is shown in Table 5.3. The numbers in parentheses are the correct digits obtained by applying the $\varepsilon$-algorithm with the same number of terms. For the entries marked by an asterisk, we used (5.3),

\section{TABLE 5.2}

Approximations to $S_{0}$ in (5.3) for $m=1, a_{1}=0, a_{0}=8$., 16., 32., using n-point quadrature in (5.4)

\begin{tabular}{r|lll}
\hline \multicolumn{1}{c}{$\mid$} & \multicolumn{1}{|c}{$a=8}$. & \multicolumn{1}{c}{$a=16}$. & \multicolumn{1}{c}{$a=32}$. \\
\hline 5 & .93098 & .6946 & .5097 \\
10 & .9313726 & .6949315 & .5099264 \\
15 & .931372933 & .6949317145 & .5099265169 \\
20 & .9313729340028 & .6949317146409 & .5099265170271 \\
25 & .9313729340031036 & .6949317146410454 & .5099265170272112 \\
30 & .9313729340031038714 & .6949317146410455900 & .5099265170272113479 \\
35 & .93137293400310387169 & .69493171464104559016 & .509926517027211348804 \\
40 & .93137293400310387169 & .69493171464104559016 & .509926517027211348804 \\
\hline
\end{tabular}


TABLE 5.3

Number of correct significant decimal digits in direct (and accelerated) summation of $S_{0}$ using 1000 terms

\begin{tabular}{c|ccccc}
\hline$m$ & $a=.5$ & $a=1$. & $a=2$. & $a=4$. & $a=8$. \\
\hline 1 & $2(2)$ & $1(2)$ & $2(2)$ & $2(2)$ & $1(1)$ \\
2 & $4(5)$ & $4(5)$ & $4(5)$ & $4(5)$ & $3(4)$ \\
3 & $7(9)$ & $7(9)$ & $6(8)$ & $6(8)$ & $5(7)^{*}$ \\
4 & $10(13)$ & $9(12)$ & $9(12)$ & $8(11)^{*}$ & $8(10)^{*}$ \\
5 & $14(17)$ & $13(15)$ & $11(14)$ & $11(13)^{*}$ & $10(12)^{*}$ \\
\hline
\end{tabular}

(5.4) to verify the number of correct digits. As is evident from Table 5.3, the $\varepsilon$-algorithm is only marginally effective on this particular series.

Example 5.2. $S_{1}=\sum_{k=1}^{\infty}(-1)^{k-1} k^{-1 / 2} /(k+a)^{m}$.

We now apply Gauss quadrature (obtained from the recursion coefficients in Table 2 of the Appendix) relative to the weight function $t^{-1 / 2} \varphi(t)$ to the integral in (3.7) (with $\nu=\frac{1}{2}$ ), using the same values of $a$ and $m$ as in Example 5.1. The results are similar to those in Table 5.1 of Example 5.1, except that convergence is slightly slower. Stratified summation similar to (5.3), (5.4), on the other hand, gives

$$
S_{1}=a_{0}^{-(m+1 / 2)} \sum_{\lambda=1}^{a_{0}}(-1)^{\lambda}\left\{s_{\lambda}-\frac{\left(\lambda / a_{0}\right)^{-1 / 2}}{\left(1+\left(\lambda+a_{1}\right) / a_{0}\right)^{m}}\right\},
$$

where

$$
s_{\lambda}= \begin{cases}-\sum_{\kappa=1}^{\infty} \frac{\left(\kappa+\lambda / a_{0}\right)^{-1 / 2}}{\left(\kappa+1+\left(\lambda+a_{1}\right) / a_{0}\right)^{m}} & \text { if } a_{0} \text { is even } \\ \sum_{\kappa=1}^{\infty} \frac{(-1)^{\kappa-1}\left(\kappa+\lambda / a_{0}\right)^{-1 / 2}}{\left(\kappa+1+\left(\lambda+a_{1}\right) / a_{0}\right)^{m}} & \text { if } a_{0} \text { is odd }\end{cases}
$$

that is, by (3.10) and (3.11),

$$
s_{\lambda}= \begin{cases}-\int_{0}^{\infty} t^{-1 / 2} \varepsilon(t) e^{-\left(\lambda / a_{0}\right) t} g_{m-1}\left(t ; 1+a_{1} / a_{0}, 1 / 2\right) d t, & a_{0} \text { even } \\ \int_{0}^{\infty} t^{-1 / 2} \varphi(t) \cdot t e^{-\left(\lambda / a_{0}\right) t} g_{m-1}\left(t ; 1+a_{1} / a_{0}, 1 / 2\right) d t, & a_{0} \text { odd }\end{cases}
$$

The use of (5.5), with $n$-point quadrature applied to (5.7), yields results converging at the same speed as those in Table 5.2.

Direct summation using 1000 terms yields 2-3 more correct digits than in Table 5.3 , but the $\varepsilon$-algorithm is now surprisingly effective, giving full accuracy (20 decimals) with only $23-27$ terms! 
Example 5.3. $S_{0}(b)=\sum_{k=1}^{\infty}(k+b)^{-1 / 2} /(k+b+1), 0 \leq b<1$.

This is another series of interest in P. J. Davis's work on spirals [4]. It can be readily evaluated with the help of Remark 1 in $\S 3$, taking $m=1, a=b+1$ in (3.10), and noting (5.1); one finds

$$
\begin{aligned}
S_{0}(b) & =\int_{0}^{\infty} t^{-1 / 2} \varepsilon(t) e^{-b t} g_{0}\left(t ; 1, \frac{1}{2}\right) d t \\
& =\frac{2}{\sqrt{\pi}} \int_{0}^{\infty} e^{-b t} \frac{F(\sqrt{t})}{\sqrt{t}} \cdot t^{-1 / 2} \varepsilon(t) d t
\end{aligned}
$$

Thus, $S_{0}(b)$ is the Laplace transform of the function $(F(\sqrt{t}) / \sqrt{t}) t^{-1 / 2} \varepsilon(t)$, which has a square root singularity at the origin and poles at integer multiples of $2 \pi i$. The last integral in (5.8) is easily computed by Gaussian quadrature relative to the weight function $t^{-1 / 2} \varepsilon(t)$. No more than 24 quadrature points are needed to get 14 correct significant decimal digits in the range $0<b<1$.

Example 5.4. $S_{0}=\sum_{k=1}^{\infty} k^{-1 / 2} /(k+i \alpha), \alpha>0$.

Here, $a=i \alpha, m=1$, hence

$$
S_{0}=\int_{0}^{\infty} t^{-1 / 2} \varepsilon(t) g_{0}\left(t ; i \alpha, \frac{1}{2}\right) d t
$$

Use of (5.1) and a simple change of variables gives

$$
g_{0}\left(t ; i \alpha, \frac{1}{2}\right)=\frac{i e^{-z^{2}}}{z} \operatorname{erf}(-i z), \quad z=\sqrt{i \alpha t}
$$

where erf is the error function. Letting $-i z=\frac{1}{2} \sqrt{\pi}(1-i) x$, i.e., $x=\sqrt{2 \alpha t / \pi}$, one finds (cf. [5, eq. 7.3.22])

$$
g_{0}\left(t ; i \alpha, \frac{1}{2}\right)=\sqrt{2 /(\alpha t)} e^{-i \alpha^{2} t^{2}}[C(\sqrt{2 \alpha t / \pi})+i S(\sqrt{2 \alpha t / \pi})]
$$

Here, $C(x), S(x)$ denote the Fresnel integrals [5, eqs. 7.3.1, 7.3.2]. They can be computed with an accuracy of up to 18 significant digits from the rational Chebyshev approximations provided (on microfiche cards) in [1]. Gaussian quadrature of (5.9), with $g_{0}$ given by (5.10), yields the results shown in Table 5.4. For each $n$, the first entry is the real part, the second the imaginary part of the Gauss approximation to $S_{0}$. Convergence is seen to deteriorate rapidly with increasing $\alpha$, which is to be expected in view of the highly oscillatory behavior of $g_{0}$ in (5.10) when $\alpha$ is large. The device of stratified summation, used successfully in Examples 5.1 and 5.2, however, can also be applied here, 
TABLE 5.4

n-point quadrature approximations to the integral in (3.3) with $\nu=\frac{1}{2}, m=1, a=i \alpha, \alpha=.5,1 ., 2 ., 4 ., 8$.

\begin{tabular}{r|lll}
\hline$n$ & \multicolumn{1}{|c}{$\alpha=.5$} & \multicolumn{2}{c}{$\alpha=1}$. \\
\hline 10 & 2.382181322854 & 2.006153 \\
& -.564259325223 & -.7964883 \\
20 & 2.38218132285517164 & 2.0061526552273 \\
& -.56425932522086830 & -.79648812356982 \\
40 & 2.38218132285517164 & 2.00615265522741423 \\
& -.56425932522086830 & -.79648812356984801 \\
80 & & 2.00615265522741424 \\
& & -.79648812356984802 \\
\hline \multicolumn{4}{|c}{$\alpha-1$} \\
\hline$n$ & & $\alpha=4$. & $\alpha=8$. \\
\hline 10 & 1.52 & -.91 & 1.0 \\
& -.846 & 1.10 & .3 \\
20 & 1.51822 & -.72 & -.7 \\
& -.84397 & 1.0978 & .73 \\
40 & 1.518231590364 & -.745 & -.54 \\
& -.843981047692 & 1.0976938 & .77 \\
80 & 1.51823159036615356 & -.7460348 & -.599 \\
& -.84398104769701668 &
\end{tabular}

and gives, with $\alpha_{0}=\lfloor\alpha\rfloor, \alpha=\alpha_{0}+\alpha_{1}, \alpha_{0} \geq 1,0 \leq \alpha_{1}<1$,

$$
\begin{aligned}
S_{0}=\alpha_{0}^{-3 / 2} \sum_{\lambda=1}^{\alpha_{0}}\left\{\int_{0}^{\infty} t^{-1 / 2} \varepsilon(t) e^{-\left(\lambda / \alpha_{0}\right) t} g_{0}(t\right. & \left.; i\left(1+\frac{\alpha_{1}}{\alpha_{0}}\right), \frac{1}{2}\right) d t \\
& \left.+\frac{\left(\alpha_{0} / \lambda\right)^{3 / 2}}{1+i\left(\alpha_{0}+\alpha_{1}\right) / \lambda}\right\} .
\end{aligned}
$$

Using (5.11) instead of (5.9) yields the results shown in Table 5.5.

Direct summation using 1000 terms gives $1-2$ correct decimal digits in the real part, and 4-5 in the imaginary part. The epsilon algorithm produces no more than one additional correct digit.

Example 5.5. $S_{1}=\sum_{k=1}^{\infty}(-1)^{k-1} k^{-1 / 2} /(k+i \alpha), \quad \alpha>0$.

Applying Gauss quadrature to

$$
S_{1}=\int_{0}^{\infty} t^{-1 / 2} \varphi(t) \cdot \operatorname{tg}\left(t ; i \alpha, \frac{1}{2}\right) d t
$$

with $g_{0}$ as in (5.10), or to formulae analogous to those in (5.5)-(5.7), yields results similar in quality to those in Table 5.4 (but converging at a slightly slower rate) and to those in Table 5.5. As in Example 5.2, here too, the $\varepsilon$-algorithm produces full accuracy ( 20 decimals) with as few as $25-28$ terms. 
TABLE 5.5

Approximations to $S_{0}$ for $\alpha_{1}=0, \alpha_{0}=8 ., 16 ., 32$. , using $n$-point quadrature for the integral in (5.11)

\begin{tabular}{r|lll}
\hline$n$ & \multicolumn{1}{|c}{$\alpha=8}$. & \multicolumn{1}{c}{$\alpha=16}$. & $\alpha=32$. \\
\hline 5 & .78217 & .55456 & .39250 \\
& -.6028 & -.46405 & -.34703 \\
10 & .78214786 & .554548189 & .392496065 \\
& -.60290377 & -.464094441 & -.347063784 \\
15 & .782147849839 & .554548181558 & .392496059680 \\
& -.602903762412 & -.464094436689 & -.347063781179 \\
20 & .7821478498420740 & .5545481815605363 & .3924960596818862 \\
& -.6029037624091237 & -.46409443668759260 & -.3470637811774942 \\
25 & .78214784984207490 & .55454818156053686 & .39249605968188663 \\
& -.60290376240912468 & -.46409443668759260 & -.34706378117749456 \\
30 & .78214784984207491 & .55454818156053686 & .39249605968188663 \\
& -.60290376240912469 & -.46409443668759260 & -.34706378117749456 \\
\hline
\end{tabular}

\section{ACKNOWLEDGMENT}

The stimulus to write this paper came from Professor P. J. Davis who showed the author the two series in Example 5.1 (with $a=m=1$ ) and Example 5.3 and their interesting connections with analytic properties of certain spirals. The author thanks Professor Davis for bringing these matters to his attention.

\section{APPENDIX}

Coefficients $\alpha_{k}, \beta_{k}$ in the recurrence relation (3.14) for the (monic) polynomials $\pi_{k}\left(\cdot ; w_{1}\right)$ and $\pi_{k}\left(\cdot ; w_{2}\right)$ orthogonal on $[0, \infty]$ with respect to the weight functions $w_{1}(t)=t^{-1 / 2} \varepsilon(t)$ and $w_{2}(t)=t^{-1 / 2} \varphi(t)$, where $\varepsilon$ and $\varphi$ are the Einstein and Fermi functions, respectively.

TABLE 1

Recursion coefficients for the polynomials $\left\{\pi_{k}\left(\cdot ; w_{1}\right)\right\}$

$\begin{array}{rc}k & \text { alpha }(k) \\ & \\ 0 & 0.7702686701927817973619158 \mathrm{D}+00 \\ 1 & 0.3187598556761524679366414 \mathrm{D}+01 \\ 2 & 0.5263746923045607509713590 \mathrm{D}+01 \\ 3 & 0.7301718513979321228472401 \mathrm{D}+01 \\ 4 & 0.9325649742557246403991717 \mathrm{D}+01 \\ 5 & 0.1134253380338705910700859 \mathrm{D}+02 \\ 6 & 0.1335527862000350912776166 \mathrm{D}+02 \\ 7 & 0.1536534463823498763879841 \mathrm{D}+02 \\ 8 & 0.1737355807916766756284331 \mathrm{D}+02 \\ 9 & 0.1938042671340346598781799 \mathrm{D}+02 \\ 10 & 0.2138628230757703840201835 \mathrm{D}+02 \\ 11 & 0.2339135212161886387283204 \mathrm{D}+02 \\ 12 & 0.2539579780077243186696702 \mathrm{D}+02 \\ 13 & 0.2739973790812632438336057 \mathrm{D}+02 \\ 14 & 0.2940326166032188956953619 \mathrm{D}+02 \\ 15 & 0.3140643765975902698313727 \mathrm{D}+02 \\ 16 & 0.3340931964524983754293190 \mathrm{D}+02 \\ 17 & 0.3541195039481598759588694 \mathrm{D}+02 \\ 18 & 0.3741436444410603887748966 \mathrm{D}+02\end{array}$
$0.1024084687983407303423387 \mathrm{D}+01$ $0.4414290426470050351659216 \mathrm{D}+01$ $0.9811302569554005490176024 \mathrm{D}+01$ $0.172 .2019846597339069655335 \mathrm{D}+02$ $0.2663816927777788994670644 \mathrm{D}+02$ $0.3806308145171306188997379 D+02$ $0.5149347226123903980540211 D+02$ $0.6692831418597948517773595 \mathrm{D}+02$ $0.8436685949264783306411561 \mathrm{D}+02$ $0.1038085462169082643494963 D+03$ $0.1252529402397974594712127 \mathrm{D}+03$ $0.1486996983438284336506672 \mathrm{D}+03$ $0.1741485438093782211156081 D+03$ $0.2015992497717325190612671 D+03$ $0.2310516275456567160834720 \mathrm{D}+03$ $0.2625055182290468743398986 \mathrm{D}+03$ $0.2959607865315635323564955 \mathrm{D}+03$ $0.3314173161504727690082355 D+03$ 
TABLE 1 (continued)

alpha (k)

beta (k)
$0.3941659002326358545261533 D+02$ $0.4141865046476185674181497 \mathrm{D}+02$ $0.4342056524499126877695173 D+02$ $0.4542235076717458307459872 \mathrm{D}+02$ $0.4742402095828609425140212 \mathrm{D}+02$ $0.4942558773005697744213263 D+02$ $0.51427 .06133920165074336592 \mathrm{D}+02$ $0.5342845067191693406019381 D+02$ $0.5542976347078243245985949 \mathrm{D}+02$ $0.5743100651735930722894178 D+02$ $0.5943218578036291651196756 \mathrm{D}+02$ $0.6143330653682819018270684 \mathrm{D}+02$ $0.6343437347190052879698644 \mathrm{D}+02$ $0.6543539076157123835591452 \mathrm{D}+02$ $0.6743636214169962577457229 D+02$ $0.6943729096593013782020592 \mathrm{D}+02$ $0.7143818025455660051707970 \mathrm{D}+02$ $0.7343903273596007722021081 D+02$ $0.7543985088191866566285380 \mathrm{D}+02$ $0.7744063693783245861012171 D+02$ $0.7944139294870716237315267 \mathrm{D}+02$ $0.8144212078158240432361167 \mathrm{D}+02$ $0.8344282214496580884979774 \mathrm{D}+02$ $0.8544349860573415523347228 D+02$ $0.8744415160388280745235353 \mathrm{D}+02$ $0.8944478246543989980858056 \mathrm{D}+02$ $0.9144539241380923082558384 \mathrm{D}+02$ $0.9344598257976295593027248 \mathrm{D}+02$ $0.9544655401027002965138026 \mathrm{D}+02$ $0.9744710767631740756087072 \mathrm{D}+02$ $0.9944764447985707932740654 \mathrm{D}+02$ $0.1014481652599921200627757 \mathrm{D}+03$ $0.1034486707984983649654826 \mathrm{D}+03$ $0.1054491618247644309211105 \mathrm{D}+03$ $0.1074496390202211457406775 \mathrm{D}+03$ $0.1094501030223216121502807 \mathrm{D}+03$ $0.1114505544281248145500172 \mathrm{D}+03$ $0.1134509937975286158636801 D+03$ $0.1154514216561919804529214 \mathrm{D}+03$ $0.1174518384981811257482317 \mathrm{D}+03$ $0.1194522447883699090278243 D+03$ $0.1214526409646209803150755 \mathrm{D}+03$ $0.1234530274397709812915552 \mathrm{D}+03$ $0.1254534046034402633346034 \mathrm{D}+03$ $0.1274537728236851684953421 \mathrm{D}+03$ $0.1294541324485088095701936 \mathrm{D}+03$ $0.1314544838072444525197788 \mathrm{D}+03$ $0.1334548272118240069215274 \mathrm{D}+03$ $0.1354551629579427346365327 \mathrm{D}+03$ $0.1374554913261300652800063 D+03$ $0.1394558125827353355323221 \mathrm{D}+03$ $0.1414561269808363275097370 \mathrm{D}+03$ $0.1434564347610776520206229 D+03$ $0.1454567361524452907721167 D+03$ $0.1474570313729829647931184 \mathrm{D}+03$ $0.1494573206304554236251909 \mathrm{D}+03$ $0.1514576041229632418445929 D+03$ $0.1534578820395132581474497 \mathrm{D}+03$ $0.1554581545605483905844150 \mathrm{D}+03$ $0.1574584218584402035356527 \mathrm{D}+03$ $0.1594586840979472824362573 D+03$
$0.3688750062461283923489100 D+03$ $0.4083337687143836916404452 \mathrm{D}+03$ $0.4497935260467352320334015 \mathrm{D}+03$ $0.4932542096307912490269402 D+03$ $0.5387157583853556378504431 D+03$ $0.5861781176531028679584024 \mathrm{D}+03$ $0.6356412382938987377235091 D+03$ $0.6871050759361041370967609 D+03$ $0.7405695903535074374070377 \mathrm{D}+03$ $0.7960347449430722040682676 \mathrm{D}+03$ $0.8535005062842724271206002 \mathrm{D}+03$ $0.9129668437649722603086484 \mathrm{D}+03$ $0.9744337292619761553875038 D+03$ $0.1037901136866798751510073 D+04$ $0.1103369042649074443403161 D+04$ $0.1170837424451482838994380 \mathrm{D}+04$ $0.1240306261711209254502793 \mathrm{D}+04$ $0.1311775535303863214785656 \mathrm{D}+04$ $0.1385245227406497752690127 D+04$ $0.1460715321376949438121714 \mathrm{D}+04$ $0.1538185801647184729491338 D+04$ $0.1617656653628716205091856 \mathrm{D}+04$ $0.1699127863628460750692831 D+04$ $0.1782599418773664961436173 D+04$ $0.1868071306944731842565880 D+04$ $0.1955543516714955967100250 D+04$ $0.2045016037296318349343018 \mathrm{D}+04$ $0.2136488858490612796066675 \mathrm{D}+04$ $0.2229961970645276686949499 D+04$ $0.2325435364613384446039056 \mathrm{D}+04$ $0.2422909031717334162190448 D+04$ $0.2522382963715819136786609 \mathrm{D}+04$ $0.2623857152773728401369017 \mathrm{D}+04$ $0.2727331591434664942211447 \mathrm{D}+04$ $0.2832806272595808712138972 \mathrm{D}+04$ $0.2940281189484884505625506 \mathrm{D}+04$ $0.3049756335639023251021868 D+04$ $0.3161231704885329922055971 D+04$ $0.3274707291322992662751263 D+04$ $0.3390183089306786334647040 D+04$ $0.3507659093431839934290477 D+04$ $0.3627135298519551530208766 \mathrm{D}+04$ $0.3748611699604546816971876 \mathrm{D}+04$ $0.3872088291922588320710826 \mathrm{D}+04$ $0.3997565070899351920256672 D+04$ $0.4125042032139995845116965 \mathrm{D}+04$ $0.4254519171419454824467366 \mathrm{D}+04$ $0.4385996484673398717394194 \mathrm{D}+04$ $0.4519473967989800862872556 \mathrm{D}+04$ $0.4654951617601066642205789 \mathrm{D}+04$ $0.4792429429876677427729711 D+04$ $0.4931907401316309269335963 \mathrm{D}+04$ $0.5073385528543389405254518 \mathrm{D}+04$ $0.5216863808299057028014556 \mathrm{D}+04$ $0.5362342237436497736185135 D+04$ $0.5509820812915623797118366 \mathrm{D}+04$ $0.5659299531798074770164711 D+04$ $0.5810778391242515224040107 \mathrm{D}+04$ $0.5964257388500208252780461 \mathrm{D}+04$ $0.6119736520910845275356023 \mathrm{D}+04$ $0.6277215785898614215056571 D+04$ 


\author{
TABLE 2 \\ Recursion coefficients for the polynomials $\left\{\pi_{k}\left(\cdot ; w_{2}\right)\right\}$
}

alpha (k)

$0.6324588697185093623661046 \mathrm{D}+00$ $0.2618492484147360028201222 \mathrm{D}+01$ $0.4579564963641043610171703 \mathrm{D}+01$ $0.6564030779299476746087191 \mathrm{D}+01$ $0.8555069923101791491989842 \mathrm{D}+01$ $0.1054905551208604898429030 \mathrm{D}+02$ $0.1254466067583060408401432 \mathrm{D}+02$ $0.1454126841626332776533809 D+02$ $0.1653854758410777936978112 \mathrm{D}+02$ $0.1853630238902031385655892 \mathrm{D}+02$ $0.2053440866145723808122330 D+02$ $0.2253278334043201314293342 \mathrm{D}+02$ $0.2453136848117392119444544 \mathrm{D}+02$ $0.2653012226419184859211550 \mathrm{D}+02$ $0.2852901364926778224060180 \mathrm{D}+02$ $0.3052801904751258293005461 \mathrm{D}+02$ $0.3252712016881291968149760 \mathrm{D}+02$ $0.3452630258357236264912560 \mathrm{D}+02$ $0.3652555473451833018142980 \mathrm{D}+02$ $0.3852486724106963523121097 \mathrm{D}+02$ $0.4052423239911886032854784 \mathrm{D}+02$ $0.4252364381449926929769257 \mathrm{D}+02$ $0.4452309612986561756291508 \mathrm{D}+02$ $0.4652258481809677698689649 \mathrm{D}+02$ $0.4852210602388269609628421 \mathrm{D}+02$ $0.5052165644075394216094150 \mathrm{D}+02$ $0.5252123321454810195841400 \mathrm{D}+02$ $0.5452083386684847465695719 \mathrm{D}+02$ $0.5652045623368841980826095 \mathrm{D}+02$ $0.5852009841604980400440341 D+02$ $0.6051975873956415655717186 \mathrm{D}+02$ $0.6251943572146066221850836 \mathrm{D}+02$ $0.6451912804326958639256424 \mathrm{D}+02$ $0.6651883452813302280453478 \mathrm{D}+02$ $0.6851855412183125570209516 \mathrm{D}+02$ $0.7051828587682640946987237 \mathrm{D}+02$ $0.7251802893877224443308380 \mathrm{D}+02$ $0.7451778253505194512639873 \mathrm{D}+02$ $0.7651754596499318109446470 \mathrm{D}+02$ $0.7851731859147789236506330 \mathrm{D}+02$ $0.80517099833717 / 8497355304 \mathrm{D}+02$ $0.8251688916100884308951775 \mathrm{D}+02$ $0.8451668608731183487411340 \mathrm{D}+02$ $0.8651649016653273904289527 \mathrm{D}+02$ $0.8851630098839871314378399 D+02$ $0.9051611817484278269255930 \mathrm{D}+02$ $0.9251594137682471448048561 D+02$ $0.9451577027152721523962350 \mathrm{D}+02$ $0.9651560455987618875274102 \mathrm{D}+02$ $0.9851544396434169830412752 D+02$ $0.1005152882269828385903095 D+03$ $0.1025151371077051766150250 \mathrm{D}+03$ $0.1045149903827039775922085 D+03$ $0.1065148478430702518687758 \mathrm{D}+03$ $0.1085147092935398729290198 D+03$ $0.1105145745513687301106930 \mathrm{D}+03$ $0.1125144434453191782850635 D+03$ $0.1145143158147450000930684 \mathrm{D}+03$ $0.1165141915087637613485474 \mathrm{D}+03$ $0.1185140703855068636699258 \mathrm{D}+03$ $\operatorname{beta}(k)$

$0.1072154929940191339530897 \mathrm{D}+01$ $0.6752170963175943015639712 \mathrm{D}+00$ $0.3167583560258398238821178 \mathrm{D}+01$ $0.7696159230518900819569277 \mathrm{D}+01$ $0.1422284065830645961871458 \mathrm{D}+02$ $0.2274710395677869363743930 \mathrm{D}+02$ $0.3326936386778514311350517 \mathrm{D}+02$ $0.4579000339917602081974728 \mathrm{D}+02$ $0.6030931435273240849088733 \mathrm{D}+02$ $0.7682751599549647344840230 \mathrm{D}+02$ $0.9534477556959725017684018 D+02$ $0.1158612232118805656707516 \mathrm{D}+03$ $0.1383769621807920822429557 \mathrm{D}+03$ $0.1628920758679360137987405 \mathrm{D}+03$ $0.1894066326819196264761766 \mathrm{D}+03$ $0.2179206895182090910153207 \mathrm{D}+03$ $0.2484342942741886876099256 \mathrm{D}+03$ $0.2809474877069469776593879 \mathrm{D}+03$ $0.3154603048296664459865959 D+03$ $0.3519727759780056334528845 \mathrm{D}+03$ $0.3904849276362956159680060 \mathrm{D}+03$ $0.4309967830860900405116312 \mathrm{D}+03$ $0.4735083629213687739354546 \mathrm{D}+03$ $0.5180196854622827391158301 D+03$ $0.5645307670907364429349043 D+03$ $0.6130416225250636069044409 D+03$ $0.6635522650467400693493852 \mathrm{D}+03$ $0.7160627066889587821461353 \mathrm{D}+03$ $0.7705729583946056944673543 \mathrm{D}+03$ $0.8270830301494799173258353 \mathrm{D}+03$ $0.8855929310953301741134029 D+03$ $0.9461026696263162082092677 \mathrm{D}+03$ $0.1008612253471766812206150 \mathrm{D}+04$ $0.1073121689767537171298761 D+04$ $0.1139630985117825228888483 D+04$ $0.1208140145648959135593239 \mathrm{D}+04$ $0.1278649177056392968932633 \mathrm{D}+04$ $0.1351158084645929041756438 \mathrm{D}+04$ $0.1425666873370009681906469 \mathrm{D}+04$ $0.1502175547859779860352491 D+04$ $0.1580684112453507229192462 \mathrm{D}+04$ $0.1661192571221852446032812 \mathrm{D}+04$ $0.1743700927990405810243678 D+04$ $0.1828209186359842875030183 D+04$ $0.1914717349723999207645930 \mathrm{D}+04$ $0.2003225421286120784146841 D+04$ $0.2093733404073509988742094 \mathrm{D}+04$ $0.2186241300950756537132996 D+04$ $0.2280749114631716813598169 D+04$ $0.2377256847690383261846901 D+04$ $0.2475764502570766919286996 \mathrm{D}+04$ $0.2576272081595900380206470 \mathrm{D}+04$ $0.2678779586976054963886758 D+04$ $0.2783287020816254278645068 \mathrm{D}+04$ $0.2889794385123156407511188 \mathrm{D}+04$ $0.2998301681811368343859689 \mathrm{D}+04$ $0.3108808912709248866969463 \mathrm{D}+04$ $0.3221316079564249594294866 \mathrm{D}+04$ $0.3335823184047838333965474 \mathrm{D}+04$ $0.3452330227760043965955907 \mathrm{D}+04$ 
TABLE 2 (continued)

\begin{abstract}
alpha (k)
$0.1205139523114388187975563 \mathrm{D}+03$ $0.1225138371607383182344134 \mathrm{D}+03$ $0.1245137248147345762237603 \mathrm{D}+03$ $0.1265136151613932056286678 D+03$ $0.1285135080948465633552084 D+03$ $0.1305134035149640899308410 \mathrm{D}+03$ $0.1325133013269586796188234 \mathrm{D}+03$ $0.1345132014410255638900482 \mathrm{D}+03$ $0.1365131037720105813681102 \mathrm{D}+03$ $0.1385130082391050492885239 \mathrm{D}+03$ $0.1405129147655647516722025 \mathrm{D}+03$ $0.1425128232784503234276992 \mathrm{D}+03$ $0.1445127337083905422629163 \mathrm{D}+03$ $0.1465126459893562457077958 \mathrm{D}+03$ $0.1485125600584607722422590 \mathrm{D}+03$ $0.1505124758557679865105743 \mathrm{D}+03$ $0.1525123933241170914867563 D+03$ $0.1545123124089595574811505 \mathrm{D}+03$ $0.1565122330582076109876996 \mathrm{D}+03$ $0.1585121552220933272468049 \mathrm{D}+03$
\end{abstract}

$\operatorname{beta}(k)$

$0.3570837212233657801133153 D+04$ $0.3691344138938122617672131 D+04$ $0.3813851009283137281105733 D+04$ $0.3938357824622001955589468 D+04$ $0.4064864586254726357105646 \mathrm{D}+04$ $0.4193371295430921239345142 D+04$ $0.4323877953352491301430689 D+04$ $0.4456384561176145930510639 \mathrm{D}+04$ $0.4590891120015742613214127 D+04$ $0.4727397630944476443598310 \mathrm{D}+04$ $0.4865904094996927900448475 \mathrm{D}+04$ $0.5006410513170979945361452 \mathrm{D}+04$ $0.5148916886429614489121902 \mathrm{D}+04$ $0.5293423215702597373710512 D+04$ $0.5439929501888060208879617 \mathrm{D}+04$ $0.5588435745853986675137462 \mathrm{D}+04$ $0.5738941948439610250053694 \mathrm{D}+04$ $0.5891448110456729724026014 \mathrm{D}+04$ $0.6045954232690948338002006 \mathrm{D}+04$ $0.6202460315902841892951491 D+04$

\section{BIBLIOGRAPHY}

1. W. J. Cody, Chebyshev approximations for the Fresnel integrals, Math. Comp. 22 (1968), 450-453. Loose microfiche suppl. A1-B4.

2. W. J. Cody, K. A. Paciorek, and H. C. Thacher, Jr., Chebyshev approximations for Dawson's integral, Math. Comp. 24 (1970), 171-178.

3. P. J. Davis, Gamma function and related functions, Handbook of Mathematical Functions, Chapter 6 (M. Abramowitz and I. A. Stegun, eds.), NBS Appl. Math. Series, vol. 55, U.S. Government Printing Office, Washington, D.C., 1964.

4. __ Spirals: From Theodorus of Cyrene to Meta-Chaos, The Hedrick Lectures, Math. Assoc. Amer., August, 1990.

5. W. Gautschi, Error function and Fresnel integrals, Handbook of Mathematical Functions, Chapter 7 (M. Abramowitz and I. A. Stegun, eds.), NBS Appl. Math. Series, vol. 55, U.S. Government Printing Office, Washington, D.C., 1964.

6. Computational aspects of orthogonal polynomials, Orthogonal Polynomials-Theory and Practice (P. Nevai, ed.), NATO ASI Series, Series C: Mathematical and Physical Sciences, vol. 294, Kluwer, Dordrecht, 1990, pp. 181-216.

7. W. Gautschi and G. V. Milovanović, Gaussian quadrature involving Einstein and Fermi functions with an application to summation of series, Math. Comp. 44 (1985), 177-190.

8. P. Henrici, Applied and computational complex analysis, vol. 1, Wiley, New York, 1984.

Department of Computer Sciences, Purdue University, West Lafayette, Indiana 47907 E-mail address:wxg@cs.purdue.edu 